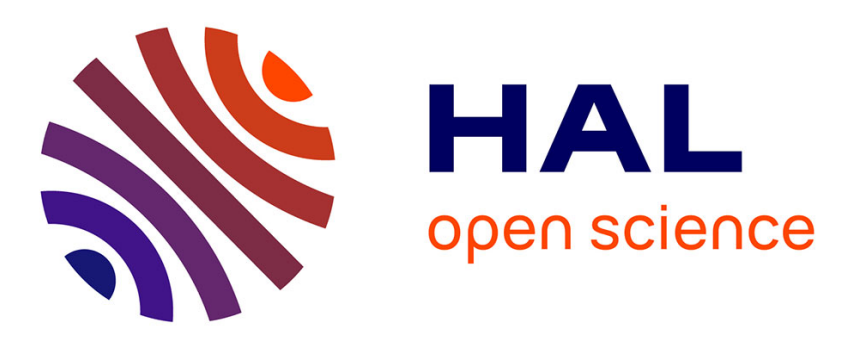

\title{
Nonlinear Control Strategies for a UAV Carrying a Load with Swing Attenuation
}

\author{
Maria Eusebia Guerrero-Sánchez, Rogelio Lozano, Pedro Castillo Garcia, \\ Omar Hernandez Gonzalez, Carlos Daniel Garcia Beltran, Guillermo \\ Valencia-Palomo
}

\section{To cite this version:}

Maria Eusebia Guerrero-Sánchez, Rogelio Lozano, Pedro Castillo Garcia, Omar Hernandez Gonzalez, Carlos Daniel Garcia Beltran, et al.. Nonlinear Control Strategies for a UAV Carrying a Load with Swing Attenuation. Applied Mathematical Modelling, 2021, 91, pp.709-722. 10.1016/j.apm.2020.09.027 . hal-02947845

\section{HAL Id: hal-02947845 \\ https://hal.science/hal-02947845}

Submitted on 16 Feb 2021

HAL is a multi-disciplinary open access archive for the deposit and dissemination of scientific research documents, whether they are published or not. The documents may come from teaching and research institutions in France or abroad, or from public or private research centers.
L'archive ouverte pluridisciplinaire HAL, est destinée au dépôt et à la diffusion de documents scientifiques de niveau recherche, publiés ou non, émanant des établissements d'enseignement et de recherche français ou étrangers, des laboratoires publics ou privés. 


\title{
Nonlinear Control Strategies for a UAV Carrying a Load with Swing Attenuation
}

\author{
M. E. Guerrero-Sánchez, R. Lozano, P. Castillo, O. Hernández-González*, , C. \\ D. García-Beltrán, G. Valencia-Palomo
}

\begin{abstract}
Two nonlinear control schemes for Unmanned Aerial Vehicles (UAV) carrying a load and their comparative results are presented in this paper. The goal is to carry the load to a desired position, with oscillation attenuation along the trajectory. The proposed control structures are hierarchical schemes consisting of nonlinear controllers to stabilize the vehicle translational movements and the payload swing together with a State-Dependent Differential Riccati Equation (SDDRE) controller to stabilize the rotational dynamics. We present new methodologies, where the nonlinear controllers are proposed to obtain precise aerial vehicle positioning and efficient load oscillation reduction. It is shown that asymptotic stability can be guaranteed by the use of the Lyapunov approach and La Salle's invariance principle. Numerical experiments were carried out to validate the nonlinear control behaviors.
\end{abstract}

Key words: Nonlinear control, Payload swing attenuation, Quadrotor, Lyapunov analysis

\section{INTRODUCTION}

Precise helicopter payload positioning with minimum swing has been researched and subject to development in recent years. Transportation of a payload suspended by a cable using Unmanned Aerial Vehicles (UAVs) is also a topic of current interest. However, this configuration has the drawback of needing an additional degree of freedom to the mathematical model in order to consider the swinging behavior of the load. Furthermore, the payload oscillation adversely affects the stability of the quadrotor. In fact, accidents can be caused by violent suspended payload swing [1], therefore, it is very important to reduce payload swing to increase safety and performance.

Some solutions have been proposed in the literature to solve the problem of transporting a hanging payload using UAVs. An example was the proposal in [2]

\footnotetext{
Email addresses: mguerrero@conacyt.mx (M. E. Guerrero-Sánchez), rlozano@hds.utc.fr (R. Lozano), pedro.castillo@hds.utc.fr (P. Castillo), ohernandezg@itesco.edu.mx (O. Hernández-González*,), cgarcia@cenidet.edu.mx (C. D. García-Beltrán), gvalencia@ith.mx (G. Valencia-Palomo)
} 
to reduce the load swing for a helicopter with a cable-attached load using input shaping. In that paper, the input shaped movements illustrate greatly-reduced residual oscillation, with only a minor increase in the displacement time. Also, a Linear Quadratic Regulator (LQR) control scheme was developed to lift and carry the payload in [3]. The nonlinear mathematical model is linearized when the UAV is flying in hover mode. Two stages for taking-off are employed: first, the aircraft takes-off without considering the load effect and then, it switches to a mode considering the load effect. An extension of this strategy is presented in [4], where an iterative LQR (iLQR) controller of a high-level dynamic mathematical model for a single aircraft carrying a payload is developed. Furthermore, in [5] the effect of dynamic disturbances on the load caused by step variations in the mass of the payload and how this affects the flight performance of helicopters and quadrotors using proportional-integral-derivative (PID) controllers is studied. Practical implementation of transit and precise positioning of loads with single or multiple UAVs are showed in [5]-[6]. In [6] the proposal is to split the system into two subsystems and use two control loops: one for the translational subsystem and one for controlling the rotation of each helicopter. In these results, the mathematical models are linearized and the controllers are developed from the linearized models.

In [7] two control strategies, a proportional-derivative (PD) controller and a sliding mode controller (SMC) with a sliding mode disturbance observer (SMDO), are tested in a quadrotor to evaluate their performance and stability under uncertain payload masses. [8] develops a nonlinear algorithm to control the aerial robot and the payload for positioning and trajectory tracking missions, nevertheless, the model is designed in two dimensions. In [9] the same control strategy is applied to two quadrotors carrying a payload attached to them by cables. [10] proposes the use of dynamic programming to generate an optimal trajectory and an adaptive controller for maneuvering without oscillation effects. Unfortunately, in these works the cable and load dynamics are neglected, they are treated as vehicle disturbances. Also, a model-based control algorithm for the translational and rotational stabilization for a quadcopter with hanging payload is introduced in [11]. However, the rotational kinetic energy of the load is neglected. A well-known technique since the 90s: Interconnection and Damping Assignment Passivity - Based Control (IDA-PBC) for an aircraft carriying a hanging load is applied in [12] and then, [13] introduces controllers with total energy-shaping. However, these algorithms need solving complicated Partial Differential Equations (PDEs) to obtain a controller. Therefore, the controllers in [12] and [13] have not a relative simplicity and intuitive design. Also, these algorithms can not be extended for the three-dimensional case, because the obtained PDEs can not be solved.

A dynamic programming-based approach that ensures oscillation-free trajectory tracking is studied in [14]. Similarly, the differentially-flat property to design trajectories is used in [16]-[17]. An adaptive controller for a flying robot carrying a load attached using a flexible cable is proposed in [18]-[19]. Here, the cable is considered as links connected in series. In [20]-[21], position tracking of the vehicle is achieved using geometric control and a mathematical model of 
the aircraft with a hanging load attached.

A method to parameterize aerial robots with a suspended payload using a complementary constraint is presented in [22]; this approach can incorporate in a nonlinear optimization problem further constraints from the quadrotor, the environment or the mission. A mathematical model and geometric controller for a quadrotor with a suspended payload through a flexible cable is presented in [23]. In [24] the attitude motion is decoupled from the system, for which a nonlinear controller is developed for the lift vector. While the vehicle's velocity and position error are converted into rotation control in order to design the translational motion control law. A control algorithm for a quadrotor based on a backstepping strategy for trajectory tracking, without being affected by the payload swing, is developed in [25]. In [26] and [27] a path tracking controller is developed based on existing Lyapunov-based path tracking control laws for freeflying aerial vehicles that are further backstepped through the vehicle rotation dynamics. A nonlinear adaptive control algorithm for the overall system which consists of an aircraft with a hanging payload has been designed under the parametric uncertainties in [28]. However, the resulting control laws include the system dynamics and are complex to implement. Finally, other relevant works regarding quadrotors control can be found in [29]-[31].

Transportation and precise positioning of a payload using a quadrotor can be analyzed similarly to the case of a crane, which has been extensively studied in the literature. For example, an extension of a Proportional Derivative (PD) control law using a coupled-dissipation nonlinear term was proposed in [32][33] in order to attenuate the load swing of an overhead crane. A nonlinear tracking controller for three-dimensional overhead cranes regardless of the initial conditions and mass variations of the load is proposed in [34]. Also, an energybased control law that ensures an improved transient response with a model-free structure is developed in [35].

As noted above, several approaches have been used to solve the control problem of a UAV carrying a load. However, few works propose nonlinear control laws that take advantage of the natural coupling between the horizontal position of the quadrotor and the payload oscillation, as it has been proposed in some studies that solve the control problem for an overhead crane.

Considering the aforementioned points, we propose a three-dimensional mathematical model and two control laws with an intuitive design for a quadrotor carrying a load. With its simple structure and less computational algorithm, the proposed strategies may be feasible for real applications. The control laws are based on a hierarchical scheme considering the well-known time-scale separation between rotational and translational dynamics of the quadrotor. Two nonlinear control strategies to control the vehicle position and the payload swing are then proposed. Our proposed controllers take advantage of the natural coupling existing between the horizontal quadcopter movement and the payload oscillation, and thus, they do not need the resolution of PDEs. This means that nonlinear feedback terms can be included in the control design for providing additional information to reduce the payload oscillation through the natural coupling between the horizontal position of the vehicle and the payload swing. The first 


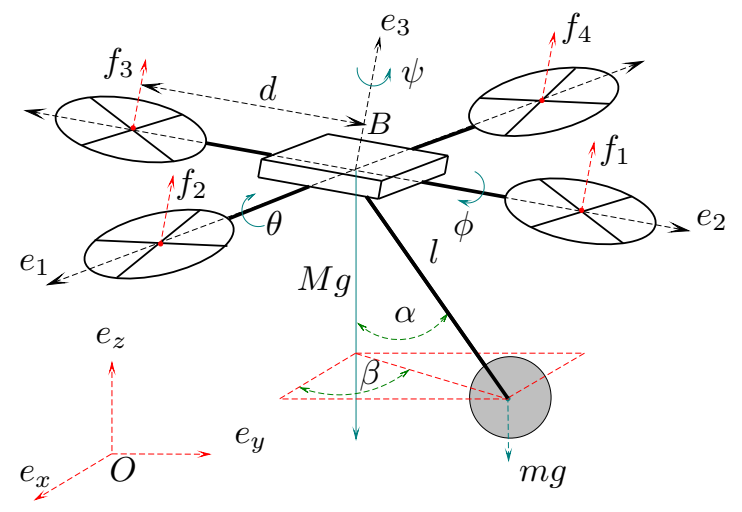

Figure 1: Quadcopter with a cable-attached load.

strategy is a PD control with a nonlinear coupled term. The second strategy is a nonlinear coupling control based on the first algorithm. Asymptotic stability of the two control laws are guaranteed by the Lyapunov technique and La Salle's invariance principle. The main contribution of this work resides in the design of these control strategies for the system consisting of a quadrotor and a cablesuspended payload, which to our best knowledge, has not yet been previously studied in the literature. The main advantage of the proposed control laws is its relatively easy and intuitive design. Moreover, it allows a state-dependent differential Riccati equation controller for the rotational dynamics to be used to perform the quadrotor stabilization. The backward integration solution method is used to solve the State-Dependent Differential Riccati Equation (SDDRE) and thus to obtain the optimal gains. The Euler-Lagrange mathematical model of the orientation dynamics is changed to form a State-Dependent Coefficient (SDC) parameterization.

The paper is structured as follows: Section 2 presents the nonlinear model of a quadcopter carrying a hanging payload; Section 3 develops two nonlinear coupling control strategies for the vehicle translational movements and the payload oscillation; Section 4 introduces a state-dependent differential Riccati equation controller to stabilize the quadrotor rotational movements; Section 5 describes numerical simulations and their corresponding results; finally, Section 6 discusses the conclusions and perspectives.

\section{MATHEMATICAL MODEL}

This section introduces the mathematical model of a quadcopter with a hanging load attached by a cable.

Some assumptions are proposed for achieving the main goal:

A1 The payload could be considered as a point-mass. 
A2 Non-aggressive maneuvers are considered, then by physical constraints $\alpha$ will move between $-90^{\circ}<\alpha<90^{\circ}$.

From Figure 1, let us consider a body fixed coordinate frame $B=\left\{e_{1}, e_{2}, e_{3}\right\}$ and an inertial coordinate frame $O=\left\{e_{x}, e_{y}, e_{z}\right\}$, fixed to the ground. The state vector is defined as $q=\left[\begin{array}{lll}\xi & \eta & \mu\end{array}\right]^{T} \in \mathbb{R}^{8}$, where $\xi \triangleq\left[\begin{array}{lll}x & y & z\end{array}\right]^{T}$ is the position of the UAV center of mass relative to $O, \eta \triangleq\left[\begin{array}{lll}\psi & \theta & \phi\end{array}\right]^{T}$ are the Euler angles, where $\theta, \phi \in(-\pi / 2, \pi / 2) . \mu \triangleq\left[\begin{array}{cc}\alpha & \beta\end{array}\right]^{T}$ defines the swing angles of the payload, $l$ represents the length of the cable and $d$ is the distance between the rotors and the gravity center. Finally, $f_{i}, i=1: 4$ introduces the thrust force provided by rotor $i$. The control input is defined as $u=\left[\begin{array}{ll}f & \tau_{\psi}\end{array}\right]^{T} \in \mathbb{R}^{4}$, where $f$ is the total thrust and $\tau=\left[\begin{array}{ccc}\tau_{\psi} & \tau_{\theta} & \tau_{\phi}\end{array}\right]^{T}$ denotes the torques.

The total kinetic energy $K(q, \dot{q})$ and the total potential energy $V(q)$ of the system can be represented as follows (more details see [13])

$$
\begin{aligned}
\left.K_{(} q, \dot{q}\right) & =\frac{1}{2} M \dot{\xi}^{T} \dot{\xi}+\frac{1}{2} \dot{\eta}^{T} J \dot{\eta}+\frac{1}{2} m \dot{\xi}_{p}^{T} \dot{\xi}_{p}+\frac{1}{2} I_{p}\left(\dot{\alpha}^{2}+\dot{\beta}^{2}\right) ; \\
V(q) & =(M+m) g z+m g l\left(1-c_{\alpha}\right) ;
\end{aligned}
$$

where $M$ is the mass of the UAV and $m$ is the mass of the load, $I_{p}$ contains the mass moments of inertia of the payload, $\xi_{p}$ is the payload position, while $J$ acts as the inertia matrix. To simplify equations, we have used the short notation $c_{\alpha}=\cos (\alpha)$.

The motion equations for a quadrotor transporting a load are obtained using the Euler-Lagrange method:

$$
\mathcal{M}(q) \ddot{q}+C(q, \dot{q}) \dot{q}+G(q)=B u ;
$$

where $\mathcal{M}(q) \in \mathbb{R}^{8 \times 8}, C(q, \dot{q}) \in \mathbb{R}^{8 \times 8}, G(q) \in \mathbb{R}^{8}, B \in \mathbb{R}^{8 \times 4}$ and are defined as follow,

$$
\begin{gathered}
\mathcal{M}(q)=\left[\begin{array}{ccc}
(m+M) \mathbf{I}_{3 \times 3} & \mathbf{0}_{3 \times 3} & \Phi_{\mu} \\
\mathbf{0}_{3 \times 3} & \Omega_{\eta} & \mathbf{0}_{3 \times 2} \\
\Phi_{\mu}^{T} & \mathbf{0}_{2 \times 3} & \Gamma_{\mu}
\end{array}\right] ; \quad C(q)=\left[\begin{array}{ccc}
\mathbf{0}_{3 \times 3} & \mathbf{0}_{3 \times 3} & C_{\mu} \\
\mathbf{0}_{3 \times 3} & C_{\eta} & \mathbf{0}_{3 \times 2} \\
\mathbf{0}_{2 \times 3} & \mathbf{0}_{2 \times 3} & \Upsilon_{\mu}
\end{array}\right] ; \\
G(q)=\left[\begin{array}{lll}
G_{z} & \mathbf{0}_{1 \times 3} & G_{\alpha}
\end{array}\right]^{T} ; \quad B(q)=\left[\begin{array}{ccc}
R e_{3} & \mathbf{0}_{1 \times 3} & \mathbf{0}_{1 \times 2} \\
\mathbf{0}_{3 \times 3} & \mathbf{I}_{3 \times 3} & \mathbf{0}_{3 \times 2}
\end{array}\right]^{T} ;
\end{gathered}
$$


with

$$
\Phi_{\mu}=\left[\begin{array}{cc}
m l c_{\alpha} c_{\beta} & -m l s_{\alpha} s_{\beta} \\
m l c_{\alpha} s_{\beta} & m l s_{\alpha} c_{\beta} \\
m l s_{\alpha} & 0
\end{array}\right] ; \quad \Omega_{\eta}=\left[\begin{array}{ccc}
I_{\psi} s_{\theta}^{2}+c_{\theta}^{2}\left(I_{\theta} s_{\phi}^{2}+I_{\phi} c_{\phi}^{2}\right) & \left(I_{\theta}-I_{\phi}\right)\left(c_{\theta} s_{\phi} c_{\phi}\right) & -I_{\psi} s_{\theta} \\
\left(I_{\theta}-I_{\phi}\right)\left(c_{\theta} s_{\phi} c_{\phi}\right) & I_{\theta} c_{\phi}^{2}+I_{\phi} s_{\phi}^{2} & 0 \\
-I_{\psi} s_{\theta} & 0 & I_{\psi}
\end{array}\right]
$$

$\Gamma_{\mu}=\left[\begin{array}{cc}m l^{2}+I_{p} & 0 \\ 0 & m l^{2} s_{\alpha}^{2}+I_{p}\end{array}\right] ; C_{\mu}=\left[\begin{array}{cc}-m l\left(c_{\alpha} s_{\beta} \dot{\beta}+s_{\alpha} c_{\beta} \dot{\alpha}\right) & -m l\left(c_{\alpha} s_{\beta} \dot{\alpha}+s_{\alpha} c_{\beta} \dot{\beta}\right) \\ m l\left(c_{\alpha} c_{\beta} \dot{\beta}-s_{\alpha} s_{\beta} \dot{\alpha}\right) & m l\left(c_{\alpha} c_{\beta} \dot{\alpha}-s_{\alpha} s_{\beta} \dot{\beta}\right) \\ m l c_{\alpha} \dot{\alpha} & 0\end{array}\right]$

$$
C_{\eta}=\left[\begin{array}{ccc}
c_{\eta_{11}} & c_{\eta_{12}} & c_{\eta_{13}} \\
c_{\eta_{21}} & c_{\eta_{22}} & c_{\eta_{23}} \\
c_{\eta_{31}} & c_{\eta_{32}} & 0
\end{array}\right] ; \quad G_{z}=\left[\begin{array}{c}
0 \\
0 \\
(M+m) g
\end{array}\right]^{T} ; \quad G_{\alpha}=\left[\begin{array}{c}
m g l s_{\alpha} \\
0
\end{array}\right]^{T}
$$

$R e_{3}=\left[\begin{array}{lll}s_{\phi} s_{\psi}+c_{\phi} c_{\psi} s_{\theta} & c_{\phi} s_{\theta} s_{\psi}-c_{\psi} s_{\phi} & c_{\theta} c_{\phi}\end{array}\right] ; \quad \Upsilon_{\mu}=\left[\begin{array}{cc}0 & -m l^{2} s_{\alpha} c_{\alpha} \dot{\beta} \\ m l^{2} s_{\alpha} c_{\alpha} \dot{\beta} & m l^{2} s_{\alpha} c_{\alpha} \dot{\alpha}\end{array}\right]$

where $R$ is the rotational matrix from the body frame to the inertial one, $c_{\eta_{11}}=$ $I_{\psi} \dot{\theta} s_{\theta} c_{\theta}+\left(I_{\theta}-I_{\phi}\right) \dot{\phi} c_{\theta}^{2} s_{\phi} c_{\phi}-I_{\theta} \dot{\theta} s_{\theta} c_{\theta} s_{\phi}^{2}-I_{\phi} \dot{\theta} s_{\theta} c_{\theta} c_{\phi}^{2}, c_{\eta_{12}}=I_{\psi} \dot{\psi} s_{\theta} c_{\theta}-\left(I_{\theta}-\right.$ $\left.I_{\phi}\right)\left(\dot{\theta} s_{\theta} c_{\phi} s_{\phi}+\dot{\phi} c_{\theta} s_{\phi}^{2}\right)+\left(I_{\theta}+I_{\phi}\right) \dot{\phi} c_{\theta} c_{\phi}^{2}-I_{\phi} \dot{\psi} s_{\theta} c_{\theta} c_{\phi}^{2}-I_{\theta} \dot{\psi} s_{\theta} c_{\theta} s_{\phi}^{2}, c_{\eta_{13}}=-I_{\psi} \dot{\theta} c_{\theta}+$ $\left(I_{\theta}-I_{\phi}\right) \dot{\psi} c_{\theta}^{2} s_{\phi} c_{\phi}, c_{\eta_{21}}=\dot{\psi} s_{\theta} c_{\theta}\left(I_{\theta} s_{\phi}^{2}+I_{\phi} c_{\phi}^{2}-I_{\psi}\right), c_{\eta_{22}}=\dot{\phi} s_{\phi} c_{\phi}\left(I_{\phi}-I_{\theta}\right), c_{\eta_{23}}=$ $I_{\psi} \dot{\psi} c_{\theta}+\left(I_{\theta}-I_{\phi}\right)\left(\dot{\psi} c_{\theta} c_{\phi}^{2}-\dot{\theta} s_{\theta} c_{\phi}-\dot{\psi} c_{\theta} s_{\phi}^{2}\right), c_{\eta_{31}}=-\dot{\psi} c_{\theta}^{2} s_{\phi} c_{\phi}\left(I_{\theta}-I_{\phi}\right)$ and $c_{\eta_{32}}=$ $\left(I_{\theta}-I_{\phi}\right)\left(\dot{\theta} s_{\phi} c_{\phi}+\dot{\psi} c_{\theta} s_{\phi}^{2}-\dot{\psi} c_{\theta} c_{\phi}^{2}\right)-I_{\psi} \dot{\psi} c_{\theta}$.

\subsection{Longitudinal dynamics}

Let us consider the longitudinal dynamics (i.e., $y=\psi=\phi=\beta=0$ ). Then, the motion equations can be written as follows

$$
\begin{aligned}
f s_{\theta} & =(M+m) \ddot{x}+m l\left(c_{\alpha} \ddot{\alpha}-s_{\alpha} \dot{\alpha}^{2}\right) ; \\
f c_{\theta} & =(M+m) \ddot{z}+m l\left(s_{\alpha} \ddot{\alpha}+c_{\alpha} \dot{\alpha}^{2}\right)+(M+m) g \\
\tau_{\theta} & =I_{\theta} \ddot{\theta} \\
0 & =m l^{2} \ddot{\alpha}+m l c_{\alpha} \ddot{x}+m l s_{\alpha} \ddot{z}+m l g s_{\alpha} .
\end{aligned}
$$

The previous equations can be expressed in the matrix form (3) as

$$
\tilde{\mathcal{M}}(q) \ddot{q}=\tilde{B} u-\tilde{C}(q, \dot{q}) \dot{q}-\tilde{G}(q) ;
$$


where

$$
\begin{aligned}
\tilde{\mathcal{M}}(q) & =\left[\begin{array}{cccc}
M+m & 0 & 0 & m l c_{\alpha} \\
0 & M+m & 0 & m l s_{\alpha} \\
0 & 0 & I_{\theta} & 0 \\
m l c_{\alpha} & m l s_{\alpha} & 0 & m l^{2}
\end{array}\right], \tilde{C}(q, \dot{q})=\left[\begin{array}{cccc}
0 & 0 & 0 & -m l s_{\alpha} \dot{\alpha} \\
0 & 0 & 0 & m l c_{\alpha} \dot{\alpha} \\
0 & 0 & 0 & 0 \\
0 & 0 & 0 & 0
\end{array}\right] \\
\tilde{G}(q) & =\left[\begin{array}{c}
0 \\
(M+m) g \\
0 \\
m l g s_{\alpha}
\end{array}\right], \tilde{B}(q)=\left[\begin{array}{cc}
s_{\theta} & 0 \\
c_{\theta} & 0 \\
0 & 1 \\
0 & 0
\end{array}\right]
\end{aligned}
$$

Notice that $\operatorname{det}(\tilde{\mathcal{M}}) \neq 0$. The translational dynamics from (14) can be rewritten as

$$
\ddot{\xi}_{x z}=\frac{1}{\operatorname{det}(\tilde{\mathcal{M}})}(D h f+N)
$$

where

$$
\begin{aligned}
\xi_{x z} & =\left[\begin{array}{ll}
x & z
\end{array}\right]^{T}, D=\left[\begin{array}{cc}
I_{\theta} l^{2} m\left(m c_{\alpha}^{2}+M\right) & I_{\theta} l^{2} m^{2} s_{\alpha} c_{\alpha} \\
I_{\theta} l^{2} m^{2} s_{\alpha} c_{\alpha} & I_{\theta} l^{2} m\left(m s_{\alpha}^{2}+M\right)
\end{array}\right]>0, \\
N & =\left[\begin{array}{ll}
n_{1} & n_{2}
\end{array}\right]^{T}, h f=\left[\begin{array}{cc}
f s_{\theta} & f c_{\theta}
\end{array}\right]^{T}, \\
\text { with } n_{1} & =-I_{\theta} l^{3} m^{2} \dot{\alpha}^{2} s_{\alpha}\left(M+2 m c_{\alpha}^{2}\right), n_{2}=-I_{\theta} l^{3} m^{2} \dot{\alpha}^{2} c_{\alpha}\left(M+2 m s_{\alpha}^{2}\right)-I_{\theta} l^{2} m M g(M+
\end{aligned}
$$
$m)$.

From the quadrotor dynamics, it is observed that the rotation dynamics is decoupled of the translational dynamics, i.e. it does not depend on the second one. Nevertheless, the translational dynamics is related with the attitude dynamics. This property allows separation into two dynamics, in general for control purposes. This implies that it is possible to have two general control schemes for this vehicle; an inner and outer control loops.

\section{NONLINEAR CONTROL STRATEGIES}

Let us decompose the three-dimensional translational dynamics into 2 twodimensional translational dynamics for UAVs, since the natural configuration of the quadrotor (rigid structure in cross) provides natural movements in the lateral or longitudinal axis, i.e., the quadrotor normally moves for simplicity either in its lateral axis or in its longitudinal axis.

Therefore, we consider first the vehicle within the longitudinal plane and then, in the lateral plane. Thus, the mathematical model (10) to (13) can be defined only by (10), (11) and (13). Moreover, (15) can be written as

$$
\ddot{\xi}_{x z}=\frac{1}{\operatorname{det}(\tilde{\mathcal{M}})}\left(D L u_{*}+N\right) ; \quad L=\left[\begin{array}{cc}
1 & 0 \\
0 & c_{\theta}
\end{array}\right] ; \quad u_{*}=\left[\begin{array}{c}
\theta_{d} \\
f
\end{array}\right]
$$


with $c_{\theta} \neq 0$ since $\theta \in(-\pi / 2, \pi / 2)$. The control objective in this work is to regulate the quadrotor position to a constant desired position denoted by $\xi_{x z d}=$ $\left[\begin{array}{ll}x_{d} & z_{d}\end{array}\right]^{T}$, i.e.,

$$
\lim _{t \rightarrow \infty} \xi_{x z}=\xi_{x z d}
$$

while at the same time reduce the payload angle $\alpha$ to zero, i.e.,

$$
\lim _{t \rightarrow \infty} \alpha=0 .
$$

\subsection{PD Control Law with Nonlinear Coupled Term}

Equation (13) can be rewritten as

$$
\ddot{\alpha}=-\frac{1}{l} \ddot{x} c_{\alpha}-\frac{\ddot{z}+g}{l} s_{\alpha} ;
$$

Note that for small angles

$$
\lim _{\alpha \rightarrow 0} \ddot{\alpha}=-\frac{1}{l} \ddot{x}-\frac{\ddot{z}+g}{l} \alpha .
$$

From the above expression, it is possible to observe that near the equilibrium point, the payload swing angle dynamics is influenced by the horizontal and vertical dynamics. Then, let $f$ be the control variable for stabilizing the vertical dynamics and $\theta_{d}$ the control variable to stabilize the vehicle translational position and the cable-suspended payload. Based on the previous, we design the following PD control law with nonlinear coupled term:

$$
u_{o}=\left[\begin{array}{c}
\theta_{d_{o}} \\
f_{o}
\end{array}\right]=u_{P D}+u_{C} ;
$$

where the " $O$ " sub-index refers to a PD control with nonlinear coupled term, $u_{P D}$ is a PD controller with gravity compensation and $u_{C}$ a nonlinear coupling term between $\dot{x}$ and $\dot{\alpha}$. These expressions are defined as

$$
u_{P D}=\left[\begin{array}{c}
-k_{p x}\left(x-x_{d}\right)-k_{v x} \dot{x} \\
\frac{-k_{p z}\left(z-z_{d}\right)-k_{v z} \dot{z}+(M+m) g}{c_{\theta}}
\end{array}\right] ; \quad u_{C}=\left[\begin{array}{c}
-k_{v \alpha} \dot{\alpha}^{2} \dot{x} \\
\frac{-k_{v \alpha} \dot{\alpha}^{2} \dot{z}}{c_{\theta}}
\end{array}\right] ;
$$

where $k_{p x}$ and $k_{p z}$ are proportional gains and the $k_{v x}, k_{v z}, k_{v \alpha}$ are used to inject damping into the system. Then, $u_{P D}$ steers the system to reach the desired horizontal and vertical positions, while $u_{C}$ provides to attenuation of the swing angle of the payload.

Theorem 3.1. The control law (21) guarantees asymptotic stability of the lateral or longitudinal quadrotor dynamics with a payload connected by a cable such that

$$
\lim _{t \rightarrow \infty}\left[\begin{array}{lll}
x & z & \alpha
\end{array}\right]=\left[\begin{array}{lll}
x_{d} & z_{d} & 0
\end{array}\right] .
$$


Proof. Now let us propose the following continuous differentiable scalar function as the Lyapunov function candidate

$$
E=H+\frac{1}{2} k_{p x}\left(x-x_{d}\right)^{2}+\frac{1}{2} k_{p z}\left(z-z_{d}\right)^{2}-(M+m) g z ;
$$

where $H=\frac{1}{2} \dot{\tilde{q}}^{T} \tilde{\mathcal{M}}(\tilde{q}) \dot{\tilde{q}}+V(\tilde{q})$ is the total energy of system and $V(\tilde{q})=$ $(M+m) g z+m g\left(1-c_{\alpha}\right)$. Then,

$$
E=\frac{1 \dot{\tilde{q}}^{T}}{2} \tilde{\mathcal{M}}(\tilde{q})+m g\left(1-c_{\alpha}\right)+\frac{1}{2} k_{p x}\left(x-x_{d}\right)^{2}+\frac{1}{2} k_{p z}\left(z-z_{d}\right)^{2} .
$$

Observe that $m g\left(1-c_{\alpha}\right) \geqslant 0$ because $-1 \leqslant c_{\alpha} \leqslant 1$. Therefore, $E$ is positive definite. Now, differentiating (23) with respect to time, we get

$$
\dot{E}=\dot{H}+k_{p x}\left(x-x_{d}\right) \dot{x}+k_{p z}\left(z-z_{d}\right) \dot{z}-(M+m) g \dot{z} .
$$

Introducing $\dot{H}$ into (24), it follows that

$\dot{E}=\dot{\tilde{q}}^{T} \tilde{\mathcal{M}}(\tilde{q}) \ddot{\tilde{q}}+\frac{1}{2} \dot{\tilde{q}}^{T} \dot{\mathcal{M}}(\tilde{q}) \dot{\tilde{q}}+\dot{\tilde{q}}^{T} \nabla_{\tilde{q}} V(\tilde{q})+k_{p x}\left(x-x_{d}\right) \dot{x}+k_{p z}\left(z-z_{d}\right) \dot{z}-(M+m) g \dot{z}$

Substituting (14) into above, it yields

$$
\begin{aligned}
\dot{E}= & -\dot{\tilde{q}}^{T} \tilde{C}(\tilde{q}, \dot{\tilde{q}}) \dot{\tilde{q}}-\dot{\tilde{q}}^{T} \tilde{G}(\tilde{q})+\dot{\tilde{q}}^{T} \tilde{B} u_{o}+\frac{1}{2} \dot{\tilde{q}}^{T} \dot{\tilde{\mathcal{M}}}(\tilde{q}) \dot{\tilde{q}}+\dot{\tilde{q}}^{T} \nabla_{\tilde{q}} V(\tilde{q}) \\
& +k_{p x}\left(x-x_{d}\right) \dot{x}+k_{p z}\left(z-z_{d}\right) \dot{z}-(M+m) g \dot{z}
\end{aligned}
$$

Taking into account that the skew-symmetric relationship $\dot{\tilde{q}}^{T}\left(\frac{1}{2} \dot{\tilde{\mathcal{M}}}(\tilde{q}) \dot{\tilde{q}}-\tilde{C}(\tilde{q}, \dot{\tilde{q}})\right) \dot{\tilde{q}}=$ 0 is satisfied and $\tilde{G}(\tilde{q})=\nabla_{\tilde{q}} V(\tilde{q})$, we obtain

$$
\dot{E}=\dot{\tilde{q}}^{T} \tilde{B} u_{o}+k_{p x}\left(x-x_{d}\right) \dot{x}+k_{p z}\left(z-z_{d}\right) \dot{z}-(M+m) g \dot{z} .
$$

Considering $y=\dot{\tilde{q}}^{T} \tilde{B}=\left[\begin{array}{ll}\dot{x} & c_{\theta} \dot{z}\end{array}\right]$ and using (21), it follows that

$$
\dot{E}=-k_{v x} \dot{x}^{2}-k_{v z} \dot{z}^{2}-k_{v \alpha} \dot{\alpha}^{2}\left(\dot{x}^{2}+\dot{z}^{2}\right) \leq 0 .
$$

Note that from $(25) \dot{E}(0)=0$. And, if $\dot{E}<0$ using the direct Lyapunov method, it could be shown that every trajectory approaches the origin. However, $\dot{E} \leq 0$ and therefore, $\dot{E}$ is negative semidefinite.

Using the La Salle's invariance principle, all the trajectories of the system converge at the equilibrium points and this implies asymptotic stability. Let $\mathrm{X}$ be the set of all points in $\Omega$ where $\dot{E}=0$. If $\dot{E}=0 \rightarrow \mathrm{X}=\{(x, z, \alpha) \mid \dot{E}(x, z, \alpha)=$ $0\} \rightarrow-k_{v x} \dot{x}^{2}-k_{v z} \dot{z}^{2}-k_{v \alpha} \dot{\alpha}^{2}\left(\dot{x}^{2}+\dot{z}^{2}\right)=0 \rightarrow \dot{\xi}_{x z}=0 \rightarrow \ddot{\xi}_{x z}=0$, which is the set $\mathrm{X}=\{(x, y, z) \mid x=$ constant $\wedge z=$ constant $\}$. We will prove that the constant $x, z$ is the equilibrium point $x=x_{d}, z=z_{d}$. Therefore, $\xi_{x z} \wedge E$ are constant. Also, if $\dot{\xi}_{x z}=0 \wedge \xi_{x z d}=\left[\begin{array}{ll}x_{d} & z_{d}\end{array}\right]^{T} \rightarrow \dot{H}=\dot{e}_{x z}=0$. Moreover, if 
$\xi_{x z}=\xi_{x z d}$ it is clear that $\theta_{d_{o}}$ from (21) is zero. However, the above expressions are satisfied even if $\dot{\alpha}=0 \vee \dot{\alpha} \neq 0$.

To complete the proof, the stability is analyzed for the case when $\dot{\alpha}=0$ and the case when $\dot{\alpha} \neq 0$. From (13) one sees that if $\ddot{\xi}_{x z}=0$ then,

$$
l \ddot{\alpha}+g s_{\alpha}=0 .
$$

Note that the above equation is satisfied if $\ddot{\alpha}=0 \wedge s_{\alpha}=0$ or $\ddot{\alpha}=-\frac{g}{l} s_{\alpha} \wedge$ $s_{\alpha} \neq 0$. Hence, from $s_{\alpha} \neq 0 \rightarrow \dot{\alpha} c_{\alpha} \neq 0 \rightarrow \ddot{\alpha} c_{\alpha}-\dot{\alpha}^{2} s_{\alpha} \neq 0$. However, from (10) one sees that if the input is zero (i.e., $\left.\theta_{d_{o}}=0\right) \wedge \ddot{\xi}_{x z}=0 \rightarrow \ddot{\alpha} c_{\alpha}-\dot{\alpha}^{2} s_{\alpha}=0$. Obviously, the above expression contradicts $s_{\alpha} \neq 0$, and hence, $\ddot{\alpha}=-\frac{g}{l} s_{\alpha} \wedge$ $s_{\alpha} \neq 0$ is not satisfied. Then, $s_{\alpha}=0$. From (26) $\ddot{\alpha}=-\frac{g}{l} s_{\alpha}$, if $s_{\alpha}=0 \rightarrow \ddot{\alpha}=0$ and $\ddot{\alpha}=0 \wedge s_{\alpha}=0$ is satisfied. Based on the above, it can be concluded that $\alpha=0$.

The conditions of the invariance principle are satisfied. Therefore, (21) guarantees asymptotic stability at the equilibrium (22). Thus, the control law (21) solves the regulation problem of the quadrotor position and leads the load angle to zero. Similar analysis can be used for the lateral dynamics.

\subsection{Nonlinear Control Law}

To improve the translational control and reduce the swing of the angle, the following nonlinear control is proposed:

$$
\begin{aligned}
u_{T}= & L^{-1}\left(-k_{p} e_{x z}-k_{v} \dot{e}_{x z}-k_{f} D^{-1} N-\frac{1}{2} k_{f} \operatorname{det}(\tilde{\mathcal{M}}) \frac{d}{d t} D^{-1} \dot{e}_{x z}\right. \\
& \left.-\frac{1}{2} k_{e} \operatorname{det}(\tilde{\mathcal{M}}) \frac{d}{d t} D^{-1}\left[\begin{array}{cc}
0 & 0 \\
1 & 0
\end{array}\right] \dot{e}_{x z}\right) /\left(k_{k}+k_{f}\right) ;
\end{aligned}
$$

where $u_{T}=\left[\begin{array}{cc}\theta_{d_{T}} & f_{T}\end{array}\right]^{T}$, the "T" sub-index refers to a nonlinear control law, $e_{x z}=\xi_{x z}-\xi_{x z d}$ defines the error vector of the quadrotor position, $k_{p}, k_{v}, k_{f}$, $k_{e}$ and $k_{k}$ are non-negative constant control gains.

The nonlinear control (27) is developed following some ideas from [33]. The control law presented in [33] is proposed for an overhead crane. In our proposal we exploit and increase the coupling between the horizontal position of the quadrotor and the load oscillation by the last term from (27).

Theorem 3.2. The system represented in (10)-(13) with the control law (27) guarantees asymptotic stability at the equilibrium (22).

Proof. Consider the following Lyapunov candidate function

$$
E=k_{k} H+\frac{1}{2} k_{f} \dot{e}_{x z}^{T}(\operatorname{det}(\tilde{\mathcal{M}})) D^{-1} \dot{e}_{x z}+\frac{1}{2} k_{p} e_{x z}^{T} e_{x z} ;
$$

which is positive definite. Then, differentiating (28) along the trajectories of the system

$$
\dot{E}=k_{k} \dot{H}+k_{f} \dot{e}_{x z}^{T}(\operatorname{det}(\tilde{\mathcal{M}})) D^{-1} \ddot{e}_{x z}+k_{p} e_{x z} \dot{e}_{x z}^{T}+\frac{1}{2} k_{f} \dot{e}_{x z}^{T}(\operatorname{det}(\tilde{\mathcal{M}})) \frac{d}{d t} D^{-1} \dot{e}_{x z}
$$


Substituting $\dot{H}$ into (29), yields

$$
\begin{aligned}
\dot{E}= & k_{k}\left(\dot{\tilde{q}}^{T} \tilde{\mathcal{M}}(\tilde{q}) \ddot{\tilde{q}}+\frac{1}{2} \dot{\tilde{q}}^{T} \dot{\tilde{\mathcal{M}}}(\tilde{q}) \dot{\tilde{q}}+\dot{\tilde{q}}^{T} \nabla_{\tilde{q}} V(\tilde{q})\right)+k_{p} e_{x z} \dot{e}_{x z}^{T} \\
& +k_{f} \dot{e}_{x z}^{T}(\operatorname{det}(\tilde{\mathcal{M}})) D^{-1} \ddot{e}_{x z}+\frac{1}{2} k_{f} \dot{e}_{x z}^{T}(\operatorname{det}(\tilde{\mathcal{M}})) \frac{d}{d t} D^{-1} \dot{e}_{x z}
\end{aligned}
$$

Introducing (14) into the above and taking into account that $\tilde{G}(\tilde{q})=\nabla_{\tilde{q}} V(\tilde{q})$ and the skew-symmetric relationship $\dot{\tilde{q}}^{T}\left(\frac{1}{2} \dot{\tilde{\mathcal{M}}}(\tilde{q}) \dot{\tilde{q}}-\tilde{C}(\tilde{q}, \dot{\tilde{q}})\right) \dot{\tilde{q}}=0$ is satisfied, we obtain

$\dot{E}=k_{k}\left(\dot{\tilde{q}}^{T} \tilde{B} u\right)+k_{f} \dot{e}_{x z}^{T}(\operatorname{det}(\tilde{\mathcal{M}})) D^{-1} \ddot{e}_{x z}+k_{p} e_{x z} \dot{e}_{x z}^{T}+\frac{1}{2} k_{f} \dot{e}_{x z}^{T}(\operatorname{det}(\tilde{\mathcal{M}})) \frac{d}{d t} D^{-1} \dot{e}_{x z}$.

Substituting (16) into the above equation, it follows that

$\dot{E}=k_{k}\left(\dot{\tilde{q}}^{T} \tilde{B} u\right)+\dot{e}_{x z}^{T}\left(k_{f} L u_{*}+k_{f} D^{-1} N+k_{p} e_{x z}+\frac{1}{2} k_{f}(\operatorname{det}(\tilde{\mathcal{M}})) \frac{d}{d t} D^{-1} \dot{e}_{x z}\right)$.

Note that $\dot{\tilde{q}}^{T} \tilde{B} u=\dot{e}_{x z}^{T} L u_{*}$ thus, we obtain the following

$$
\dot{E}=\dot{e}_{x z}^{T}\left(k_{k}+k_{f}\right) L u_{*}+k_{f} D^{-1} N+\frac{1}{2} k_{f} \operatorname{det}(\tilde{\mathcal{M}}) \frac{d}{d t} D^{-1} \dot{e}_{x z}+k_{p} e_{x z}
$$

Finally, using (27) into $u_{*}$, we get

$$
\dot{E}=-k_{v}\left(\dot{e}_{x z}^{T} \dot{e}_{x z}+\dot{e}_{x z}^{T} \operatorname{det}(\tilde{\mathcal{M}}) \dot{N}\left[\begin{array}{cc}
0 & 0 \\
1 & 0
\end{array}\right] \dot{e}_{x z}\right) \leq 0
$$

The proposed control law guarantees the regulation of the UAV to a desired position and simultaneously damped the payload oscillation. The controller for the lateral plane can be obtained in a similar way.

\section{SDDRE CONTROLLER}

A nonlinear SDDRE control strategy for the attitude subsystem is developed.

\subsection{SDDRE background}

Consider the nonlinear system expressed as follows

$$
\begin{aligned}
& \dot{x}(t)=f(x(t), t)+g(x(t), u(t), t) ; \\
& y(t)=h(x(t), t) ;
\end{aligned}
$$

where $x(t) \in \mathbb{R}^{n}, f(x(t), t)$ and $g(x(t), u(t), t)$ are smooth functions, the equilibrium point is $f(0, t)=0$. The cost function to be minimized is given by:

$$
J=\frac{1}{2} \int_{0}^{\infty}\left(x^{T}(t) Q(x(t)) x(t)+u^{T}(t) R(x(t)) u(t)\right) d t ;
$$


where the state and input weighting matrices are assumed state-dependent such that $Q=Q^{T}, Q \geqslant 0$ and $R=R^{T}, R>0$. The state-dependent coefficient parameterization needs to be used to form:

$$
\begin{aligned}
& f(x(t), t)=A(x(t), t) x(t) ; \\
& g(x(t), u(t), t)=B(x(t), t) u(t) ; \\
& h(x(t), t)=C(x(t), t) x(t) ;
\end{aligned}
$$

where $A(x(t), t) \in \mathbb{R}^{n \times n}, B(x(t), u(t), t) \in \mathbb{R}^{n \times m}$ both depends on the state variables and $u(t) \in \mathbb{R}^{m}$. Thus, system (30) can be rewritten as follows

$$
\begin{aligned}
& \dot{x}(t)=A(x(t), t) x(t)+B(x(t), t) u(t) ; \\
& y(t)=C(x(t), t) x(t) .
\end{aligned}
$$

The SDC parameterization was realized, i.e., the variables $x(t)$ and $u(t)$ were factorized to obtain the standard form for SDDRE derivation. The pair of $(A(x(t), t), B(x(t), t))$ is completely controllable in the linear sense and the pair $(A(x(t), t), C(x(t), t))$ is completely observable in the same sense. To minimize the above cost function (30), a state optimal feedback controller can be obtained as follows

$$
u(t)=-R^{-1}(x(t), t) B^{T}(x(t), t) K(x(t), t) x(t) ;
$$

where $K$ is the positive solution of the SDDRE, which is expressed as follows [36]

$$
\begin{aligned}
\dot{K}= & -K(x(t), t) A(x(t), t)-A^{T}(x(t), t) K(x(t), t) \\
& +K B(x(t), t) R^{-1}(x(t), t) B^{T}(x(t), t) K(x(t), t)-Q(x(t), t) .
\end{aligned}
$$

Thus, (34) is a nonlinear controller with the characteristic that the linearization of the system equations is not required. The SDDRE can be solved by using backward integration, state transition matrix, Lyapunov based method, etc [37]. In this work, the SDDRE controller is solved applying the backward integration method as this method is widely used in the solution of optimal control problems with a final boundary condition.

\subsection{Rotational subsystem nonlinear SDDRE Control}

The Euler-Lagrange rotational equations of a quadrotor can be written from (3) as follows:

$$
\Omega_{\eta}(\eta) \ddot{\eta}+C_{\eta}(\eta) \dot{\eta}=\tau
$$

Remember that $\eta=\left[\begin{array}{lll}\psi & \theta & \phi\end{array}\right]^{T}$. The idea is to apply the SDDRE approach. Then, considering the state vector as $x(t)=\left[\begin{array}{ll}\eta & \dot{\eta}\end{array}\right]^{T},(36)$ can be represented as (30), with

$$
f(x(t), t)=\left[\begin{array}{c}
\dot{\eta} \\
-\Omega_{\eta}^{-1}(\eta) C_{\eta}(\eta) \dot{\eta}
\end{array}\right], \quad g(x(t), u(t), t)=\left[\begin{array}{c}
0_{3 \times 1} \\
\Omega_{\eta}^{-1}(\eta) \tau
\end{array}\right] .
$$


Thus, the SDDRE approach takes the nonlinearities of the dynamics by forcing it to a quasi-linear form employing state-dependent coefficient. Then, one can rewrite (36) as a possible set of SDC matrices:

$$
\dot{x}(t)=\left[\begin{array}{cc}
0_{3 \times 3} & I_{3 \times 3} \\
0_{3 \times 3} & -\Omega_{\eta}^{-1}(\eta) C_{\eta}(\eta)
\end{array}\right] x(t)+\left[\begin{array}{c}
0_{3 \times 3} \\
\Omega_{\eta}^{-1}(\eta)
\end{array}\right] \tau .
$$

Note that this parametrization is not unique and the existence of a solution of the SDDRE for a particular factorization of the system is not guaranteed. Even, how to precisely select the corresponding SDC matrix from all possible candidates via the presented parametrization, is worth investigating and currently under development. Also, other important aspect in the existence of the solution of the SDDRE is the selection of the $Q(x(t), t)$ and $R(x(t), t)$ weighting matrices, in general these matrices may be state-dependent.

A key aspect of this approach is to obtain a fully controllable pair $A(x(t), t)$ and $B(x(t), t)$ and the matrices $A(x(t), t)$ and $C(x(t), t)$ form a fully observable pair. Therefore, to prove the controllability of the proposed model in state space structure, the linear sense of the SDC parameterization should be verified. Then, we obtain the linear form from these matrices as:

$$
A=\left[\begin{array}{ll}
0_{3 \times 3} & I_{3 \times 3} \\
0_{3 \times 3} & 0_{3 \times 3}
\end{array}\right], \quad B=\left[\begin{array}{c}
0_{3 \times 3} \\
\operatorname{dig}\left[I_{\phi}^{-1}, I_{\theta}^{-1}, I_{\psi}^{-1}\right]_{3 \times 3}
\end{array}\right]
$$

The controllability matrix has full rank, then the system is controllable. Using the nonlinear system (37), the SDDRE (35) is then solved on-line via backward integration method to obtain the $K(x(t), t) \in \mathbb{R}^{6 \times 6}$ control gain matrix with the following final values:

$$
K=\left[\begin{array}{cccccc}
20.0342 & 0.0342 & 0 & 0 & 0 & 0 \\
0.0342 & 0.0342 & 0 & 0 & 0 & 0 \\
0 & 0 & 21.2463 & 0.0469 & 0 & 0 \\
0 & 0 & 0.0469 & 0.0664 & 0 & 0 \\
0 & 0 & 0 & 0 & 17.3611 & 0.0469 \\
0 & 0 & 0 & 0 & 0.0469 & 0.0543
\end{array}\right]
$$

Therefore, we can apply the feedback controller (34) to obtain a control scheme for the rotational subsystem stabilization.

\section{NUMERICAL SIMULATIONS AND RESULTS}

To validate the behavior of the two proposed translational controllers given by (21) and (27), some simulations have been carried out. We compare both controllers under the same conditions. In order to implement the SDDRE, the solution of the optimal gain matrix was substituted into the control law (34). The weighting matrices were selected as $R=\operatorname{dig}[1,100,100]_{3 \times 3}$ and $Q=\operatorname{dig}[20,20,15,30,15,20]_{6 \times 6}$. The simulation goal was to displace the UAV following a square of 5 meter length at 1 meter height, fastly and with reduction of the payload oscillation. 
In simulations we used model parameters close to real aerial platforms, such parameters are $M=0.4 \mathrm{Kg}, m=0.03 \mathrm{Kg}, l=0.35 \mathrm{~m}, I_{\psi}=0.3 \mathrm{gm}^{2}, I_{\theta}=I_{\phi}=$ $0.177 \mathrm{kgm}^{2}$. In the following figures the behavior of the PD control strategy with nonlinear coupled term and the nonlinear control strategy are showed.

\subsection{PD Control Law with Nonlinear Coupled Term}

Figure 2a displays the positions $x, y$ and $z$ of the aircraft during the flight test. Notice that, big changes in coordinates $x$ and $y$ are demanded and achieved in a short period of time of about $5 \mathrm{~m}$ in $7 \mathrm{~s}$. Observe also that the performance of the controller is similar in the $x$ and $y$ axis. Figure $2 \mathrm{~b}$ shows the payload swing angles $\alpha$ and $\beta$. Note that the main goal of this paper is to attenuate the swing angles while carrying the load. Here, we can see good performance of the closed-loop system, since the oscillation angles always remain well bounded and smaller than $\pm 15^{\circ}$, despite the fast maneuvers performed along the $x$ and $y$ axis. The quadrotor rotation dynamics is shown in Figure 2c, it can be noticed that the orientation controller steers the attitude of the quadrotor converge to the origin. The thrust force and torques are presented in Figure 2d. Lastly, a three-dimensional view of the way in which the quadrotor follows the set-points is displayed in Figure 2e.

\subsection{Nonlinear Control Law}

Similarly to the previous case, a second simulation has been obtained in order to show the behavior of the second developed control strategy. Figure 3a displays the positions $x, y$ and $z$ of the quadrotor during the validation. On one hand, note that the performance of the two control strategies were similar for the positions $x$ and $y$. However, a reduction of the settling time can be observed in the second control law. In this way, big changes in coordinates $x, y$ are demanded and achieved in short time, about $5 \mathrm{~m}$ in $5 \mathrm{~s}$. On the other hand, the vertical position dynamics of the two control laws are very similar as could be expected from (20). Figure 3b presents the load swing angles $\alpha$ and $\beta$. We can see that the swing angles show an overshoot maximum of $\pm 10^{\circ}$ and that the swing reduction is accomplished in almost $4 s$, regardless of the fast maneuvers performed along the $x$ and $y$ axis. The quadrotor rotation is displayed in Figure $3 \mathrm{c}$, and the inputs signals are shown in Figure 3d. Finally, a view in the threedimensional space of the quadrotor move is presented in Figure 3e, here we can observe the appropriate behavior of our controller.

\subsection{Comparison among the proposed control laws}

In summary, Figures 2a-3e show good behavior of the proposed controllers. Nevertheless, the nonlinear control law was found to have better performance than the PD control law with a nonlinear coupled term for the control objective of transporting a payload from an initial position to the goal position, with reduction of the swing. This is clear in Figures 2b, 2c, 3b and 3c. From Figures $2 \mathrm{~b}$ and $3 \mathrm{~b}$, it can be observed that the settling time and the overshoot of the payload oscillation angle obtained from control law $u_{o}$ is larger than the payload 


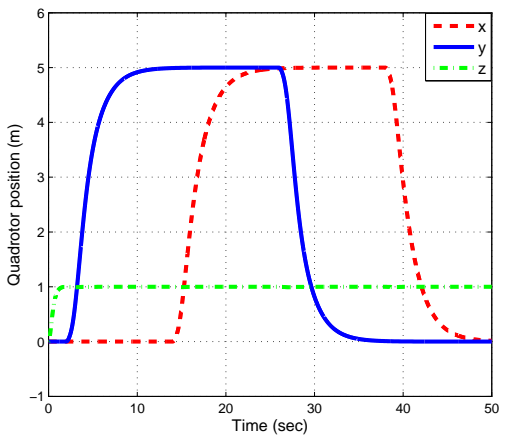

(a) Quadrotor position.

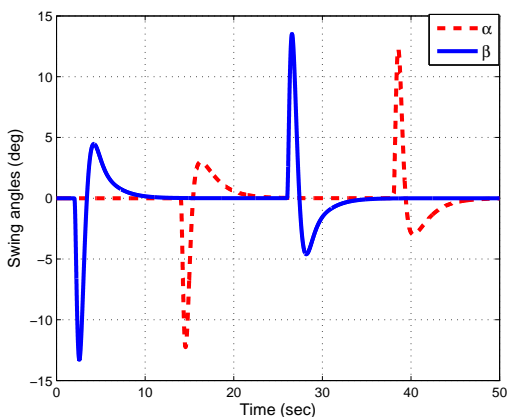

(b) Swing angles.

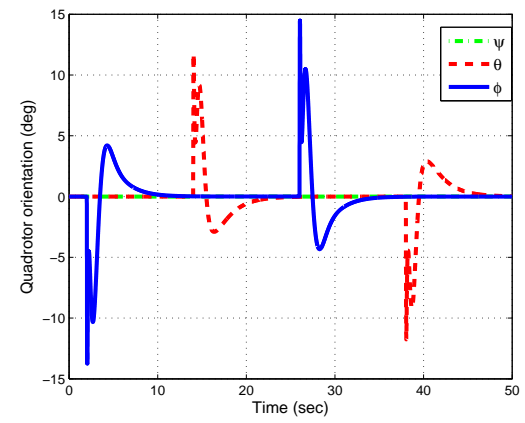

(c) Quadrotor attitude.

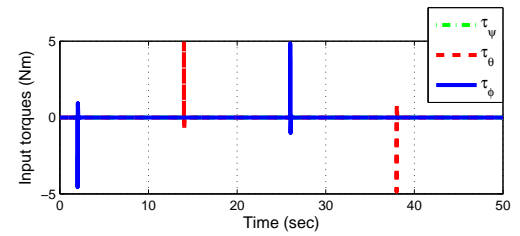

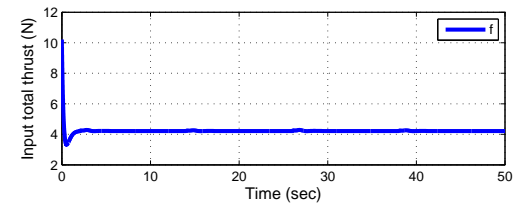

(d) Control inputs.

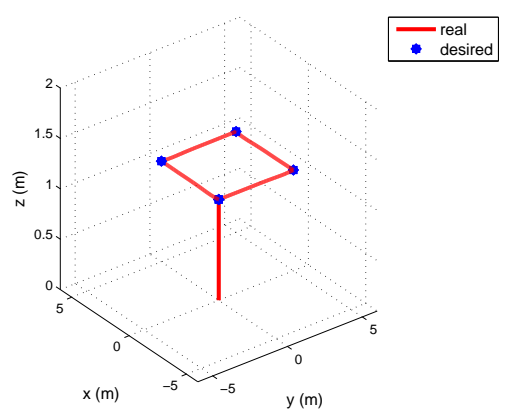

(e) Three-dimensional trajectory.

Figure 2: Simulation results for the PD control law with nonlinear coupled term. 


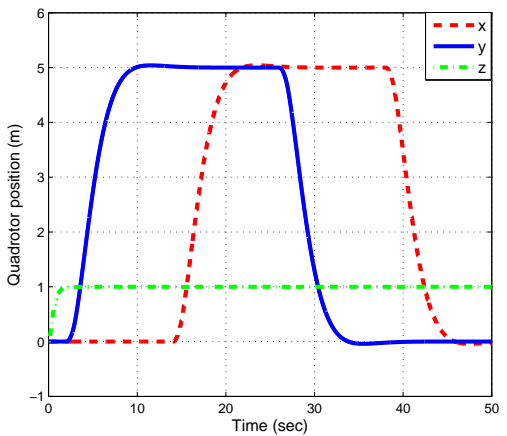

(a) Quadrotor position.

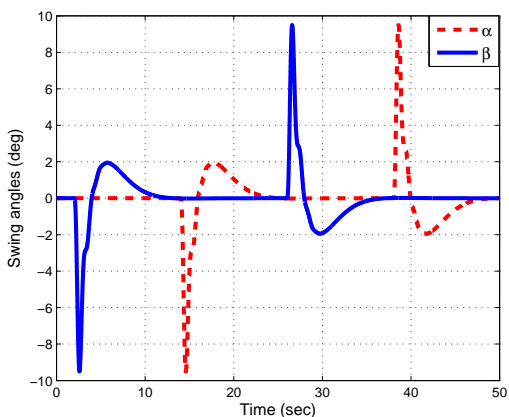

(b) Swing angles.

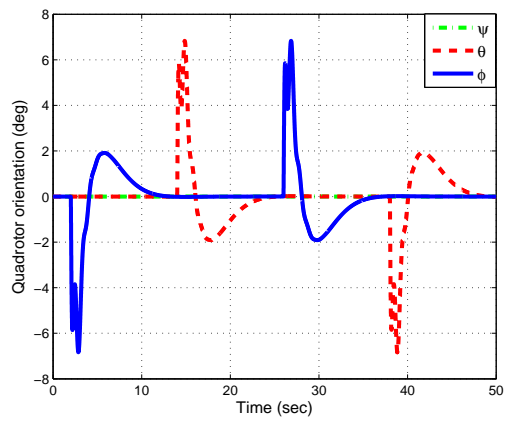

(c) Quadrotor attitude.

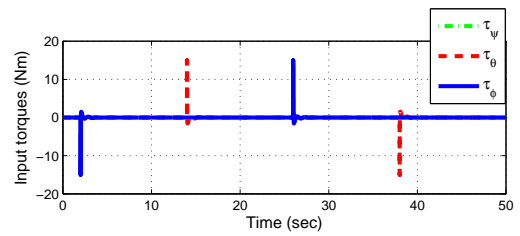

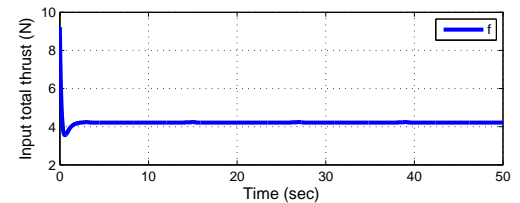

(d) Control inputs.

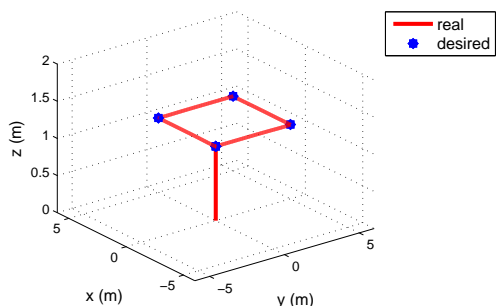

(e) Three-dimensional trajectory.

Figure 3: Simulation results for the nonlinear control law. 
swing angle obtained with the control law $u_{T}$. From Figures $2 \mathrm{c}$ and $3 \mathrm{c}$, it can be observed a similar behavior for the quadrotor attitude. Also, these figures show that the swing angles and the orientation dynamics, are quickly regulated to zero using the controller $u_{T}$. From the simulation results, we can see more advantages in the nonlinear control law, in the sense that it has better transient response.

\section{CONCLUSIONS}

This work described two novel control laws for stabilizing a quadcopter transpoting a payload attached by a cable. The proposed controllers take advantage of the natural coupling existing between the horizontal vehicle movement and the payload oscillation.

We have presented a PD control law with nonlinear coupled term and a fully nonlinear control law to reduce the oscillation of the load attached by a rod to a UAV. The main advantage of the proposed control strategies is their relative simplicity and intuitive design. The numerical results showed the good performance of the two developed control laws for the purpose of carrying a load, with swing suppression. The attitude dynamics was stabilized by a feedback control law that solves a state-dependent differential Riccati equation. Numerical experiments have shown that the performances of the proposed controllers are satisfactory. However, the nonlinear control law had better performance in the payload swing angle. Future works include extending the translational control laws for the three-dimensional case, the cable dynamic model and the variation of payload weight taken as input uncertainties.

\section{References}

[1] M. Bernard, K. Kondak, I. Maza and A. Ollero, "Autonomous Transportation and Deployment with Aerial Robots for Search and Rescue Missions", Journal of Field Robotics, vol. 28(6), pp. 914-932, 2011.

[2] J. Potter, W. Singhose and M. Costello, "Reducing Swing of Model Helicopter Sling Load Using Input Shaping", International Conference on Control and Automation (ICCA), Santiago, Chile, pp. 348-353, 2011.

[3] Y. Alothman, W. Jasim and D. Gu, "Quad-rotor Lifting-Transporting Cable-Suspended Payloads Control", 21st International Conference on Automation and Computing (ICAC), Glasgow, UK, 2015.

[4] Y. Alothman and D. Gu, "Quadrotor Transporting Cable-Suspended Load using iterative Linear Quadratic Regulator (iLQR) Optimal Control", 8th Computer Science and Electronic Engineering Conference (CEEC), Colchester, UK, 2016.

[5] P. Pounds, D. Bersak and A. Dollar, "Stability of small-scale UAV helicopters and quadrotors with added payload mass under PID control", Auton Robot, vol. 33, pp. 129-142, 2012. 
[6] M. Bernard and K. Kondak, "Generic Slung Load Transportation System Using Small Size Helicopters", IEEE International Conference on Robotics and Automation (ICRA), pp. 3258-3264, 2009.

[7] X. Zhou, R. Liu, J. Zhang and X. Zhang, "Stabilization of a Quadrotor Uncertain Suspended Load using Sliding Mode Control", International Design Engineering Technical Conferences and Computers and Information in Engineering Conference (IDETC), Charlotte, North Carolina, USA, pp. 1-8, 2016.

[8] I. H. Beloti, A. Santos and M. Sarcinelli, "Modelling and Control of a PVTOL Quadrotor Carrying a Suspended Load", International Conference on Unmanned Aircraft Systems (ICUAS), Colorado, USA, pp. 444-450, 2015 .

[9] I. H. Beloti, A. Santos and M. Sarcinelli, "Avoiding Obstacles in Cooperative Load Transportation", ISA Transaction, vol. (91), pp. 253-261, 2019.

[10] I. Palunko, P. Cruz, and R. Fierro, "Agile load transportation", IEEE Robotics and Automation Magazine, vol. (19), no. 3, pp. 69-79, 2012.

[11] S. Sadr, A. Moosavian, and P. Zarafshan, "Dynamics Modeling and Control of a Quadrotor with Swing Load", Journal of Robotics, 2014.

[12] M.E. Guerrero, D.A. Mercado, R. Lozano and C.D. García, "Passivity Based Control for a Quadrotor UAV Transporting a Cable-Suspended Payload with Minimum Swing", Conference on Decision and Control (CDC), Osaka, Japan, pp. 6718-6723, 2015.

[13] M.E. Guerrero-Sánchez, D.A. Mercado-Ravell, R. Lozano, C.D. GarcíaBeltrán, "Swing-attenuation for a quadrotor transporting a cablesuspended payload", ISA Transactions, vol. 68, pp. 433-449, 2017.

[14] I. Palunko, R. Fierro and P. Cruz, "Trajectory Generation for Swing-Free Maneuvers of a Quadrotor with Suspended Payload: A Dynamic Programming Approach", IEEE International Conference on Robotics and Automation, Saint Paul, Minnesota, USA, pp. 2691-2697, 2012.

[15] K. Macedo-Cabral, S.R. Barros, S.N. Givigi and C.L. Nascimento, "Design of model predictive control via learning automata for a single UAV load transportation", Systems Conference (SysCon) 2017 Annual IEEE International, pp. 1-7, 2017.

[16] K. Sreenath, T. Lee and V. Kumar, "Geometric Control and Differential Flatness of a Quadrotor UAV with a Cable-Suspended Load", Conference on Decision and Control, pp. 2269-2274, 2013.

[17] K. Sreenath, N. Michael and V. Kumar, "Trajectory Generation and Control of a Quadrotor with a Cable-Suspended Load A Differentially-Flat Hybrid System", IEEE International Conference on Robotics and Automation (ICRA), Karlsruhe, Germany, pp. 4873-4880, 2013. 
[18] S. Dai, T. Lee and D.S. Bernstein, "Adaptive Control of a Quadrotor UAV Transporting a Cable-Suspended load with Unknown Mass", Conference on Decision and Control, pp. 15-17, 2014.

[19] F.A. Goodarzi, D. Lee and T. Lee, "Geometric Control of a Quadrotor UAV Transporting a Payload Connected via Flexible Cable", IEEE International Journal of Control, Automation and Systems, vol. (13), no. 6, pp. 1-13, 2015.

[20] F.A. Goodarzi, D. Lee and T. Lee, "Geometric Stabilization of a Quadrotor UAV with a Payload Connected by Flexible Cable", American Control Conference (ACC), Portland, Oregon, USA, pp. 4925-4930, 2014.

[21] P. Cruz and R. Fierro, "Autonomous Lift of a Cable-suspended Load by an Unmanned Aerial Robot", IEEE Conference on Control Applications (CCA), Antibes, France, pp. 802-807, 2014.

[22] P. Foehn, D. Falanga, N. Kuppuswamy, R. Tedrake and D. Scaramuzza, "Fast Trajectory Optimization for Agile Quadrotor Maneuvers with a Cable-Suspended Payload", Robotics Science and Systems, Cambridge, Massachusetts, USA, pp. 1-10, 2017.

[23] P. Kotaru, G. Wu and K. Sreenath, "Dynamics and Control of a Quadrotor with a Payload Suspended through an Elastic Cable", American Control Conference (ACC), Seattle, WA, USA, to appear, 2017.

[24] L. Qian and H.T. Liu, "Dynamics and Control of A Quadrotor with A Cable Suspended Payload", 30th Canadian Conference on Electrical and Computer Engineering (CCECE), Ontario, Canada, 2017.

[25] K. Klausen, T.I. Fossen and T.A. Johansen, "Nonlinear Control of a Multirotor UAV with Suspended Load", IEEE International Conference on Unmanned Aircraft Systems (ICUAS), Denver, USA, pp. 176-184, 2015.

[26] D. Cabecinha, R. Cunha and C. Silvestre, "A trajectory tracking control law for a quadrotor with slung load", Automatica, vol. (106), pp. 384-389, 2019.

[27] G. Yu, D. Cabecinhas, R. Cunha and C. J. Silvestre, "onlinear backstepping control of a quadrotor slung load system", IEEE/ASME Transactions on Mechatronics, 2019.

[28] S. Yang and B. Xian, "Energy-based Nonlinear Adaptive Control Design for the Quadrotor UAV System with a Suspended Payload", IEEE Transactions on Industrial Electronics, 2019.

[29] Z. Xu, X. Nian, H. Wang and Y. Chen, "Robust guaranteed cost tracking control of quadrotor UAV with uncertainties ", ISA Trans, vol. 69, pp. 157-165 2017. 
[30] O. Mofid and S. Mobayen, "Adaptive sliding mod control for finite-time stability of quad-rotor UAVs with parametric uncertainties", ISA Trans, vol. 72 , pp. 1-14, 2018.

[31] W. Cai, J. She, M. Wu and Y. Ohyama, "Disturbance suppression for quadrotor UAV using sliding-mode-observer-based equivalent-inputdisturbance approach", ISA Transactions, vol. 92, pp. 286-297, 2019.

[32] G. C. Konstantopoulos and A. T. Alexandridis, "Simple energy based controllers with nonlinear coupled-dissipation terms for overhead crane systems", Conference on Decision and Control (CDC), Shanghai, China, pp. 3149-3154, 2009.

[33] Y. Fang, W. E. Dixon, D. M. Dawson, and E. Zergeroglu, "Nonlinear Coupling Control Laws for an Underactuated Overhead Crane System", IEEE/ASME Transactions on Mechatronics, vol. 8, no. 3, 2003.

[34] D. Chwa, "Nonlinear Tracking Control of 3-D Overhead Cranes Against the Initial Swing Angle and the Variation of Payload Weight", IEEE Transactions on Control Systems Technology, vol. 17, no. 4, 2009.

[35] N. Sun and Y. Fang, "New Energy Analytical Results for the Regulation of Underactuated Overhead Cranes: An End-Effector Motion-Based Approach", IEEE Transactions on Industrial Electronics, vol. 59, no. 12, 2012.

[36] M.H. Korayem and S.R. Nekoo, "Finite-time state-dependent Riccati equation for time-varying nonaffi ne systems: Rigid and flexible joint manipulator control", ISA Transactions, pp. 125-144, 2015.

[37] T. Nguyen and Z. Gajic, "Solving the Matrix Differential Riccati Equation: A Lyapunov Equation Approach", IEEE Transactions on Automatic Control (TAC), pp. 191-194, 2010. 